\begin{tabular}{|c|} 
Syphilis \\
symposium
\end{tabular}

\section{The great pretender returns to Dublin, Ireland}

\author{
S Hopkins, F Lyons, F Mulcahy, C Bergin
}

Background: An outbreak of syphilis has been identified primarily in Dublin, Ireland. On a background of a stable low incidence of syphilis, a significant increase in the number of cases of syphilis has been observed over the past 18 months.

Results: 181 cases of syphilis were seen in this period. 121 were defined as early syphilis. These cases were mainly among men who have sex with men $(92 \%)$. A high rate of HIV co-infection was present; 16 patients who regularly attended HIV clinics were diagnosed with early syphilis. Nine patients were co-diagnosed with HIV and early syphilis. High risk behaviour and concurrent diagnoses with other sexually transmitted infections were prevalent in this cohort. Sexual networks were also investigated.

Conclusion: An education campaign, widespread advertisements, and on-site testing in commercial venues have revealed further cases. Safer sex messages need to be emphasised particularly among the HIV population. Further innovative strategies continue to be explored. (Sex Transm Inf 2001;77:316-318)

Keywords: syphilis; epidemiology; Dublin

\section{Introduction}

St James's Hospital, James's Street, Dublin 8, and Gay Men's Health Project, Haddington Road, Dublin 2, Ireland S Hopkins F Lyons

C Bergin

Correspondence to: Dr S Hopkins, Department of Genitourinary Medicine and Infectious Diseases, St James's Hospital, James's Street, Dublin 8, Ireland 29 August 2001
F Mulcahy fmulcahy@stjames.ie

Accepted for publication
The developed world had seen relatively low rates of syphilis throughout the early 1990s. However, over the past 2 years outbreaks of primary and secondary syphilis have been reported in Brighton, ${ }^{1}$ Manchester, ${ }^{2}$ Seattle, ${ }^{3}$ and southern California. ${ }^{4}$

From January 2000 to June 2001, 181 new cases of syphilis were diagnosed at our clinics in St James's Hospital and the Gay Men's Health Project (GMHP) - a community based sexual health clinic for homosexual men. This was against a background of a low incidence of infection in the mid-1990s (fig 1). These clinics represent the largest STI services in Ireland seeing over 25000 new patients per year. This rising incidence is of concern for current sexual

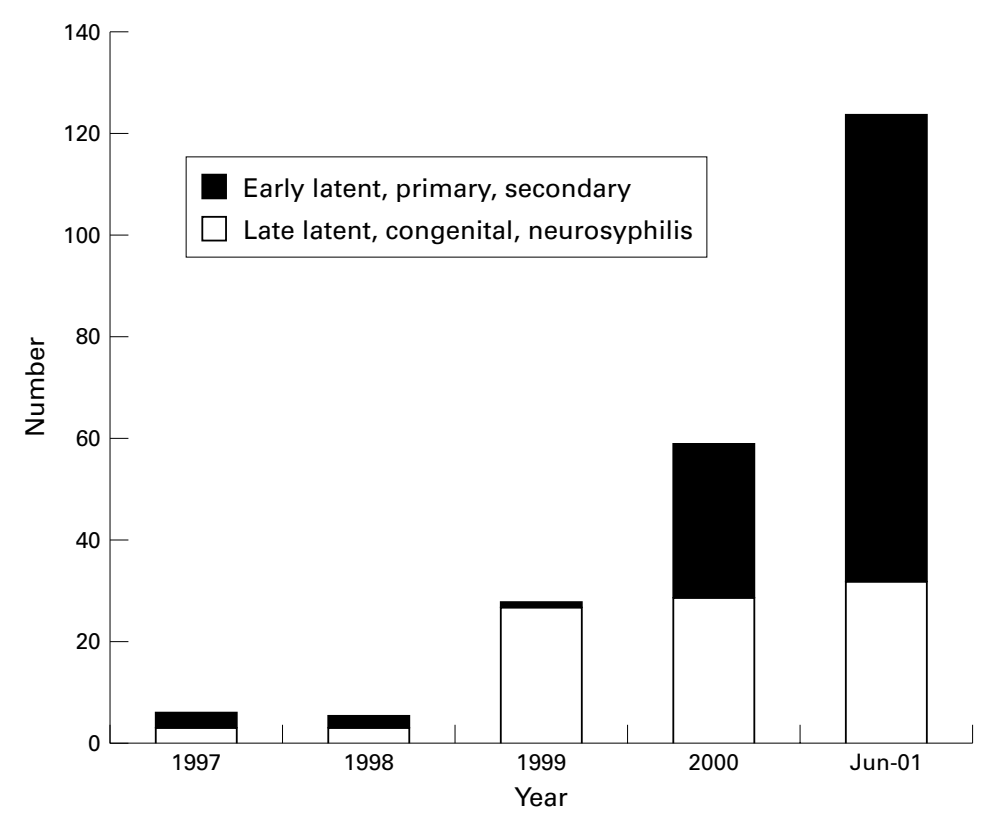

Figure 1 Epidemiology of syphilis attending clinic 1997-2001. health practices, the long term sequelae of syphilis, and the increased risk of HIV acquisition.

\section{Methods}

In October 2000, given a sustained increase in the number of cases diagnosed, an outbreak committee was initiated with members representing the medical staff of St James's Hospital and the GMHP, National Disease Surveillance centre (NDSC), public health, and members of the homosexual community (fig 2).

Demographics were recorded in all cases including age, sex, nationality, and sexual orientation. In addition, all cases of early syphilis had a structured interview performed by a health adviser and a doctor. In these 121 cases, further details pertaining to numbers of sexual contacts in the past 3 months and 1 year and risk behaviour (drug and alcohol use, condom use) were recorded. Sexual and social networks (bars, clubs, saunas, commercial sex workers, internet, abroad) and history of sexually transmitted infections including HIV were identified.

\section{Results}

From January 2000 to June 2001, 181 cases were reported. Sixty cases were latent infection. One hundred and twenty one cases of early infectious syphilis were identified. Of these cases, 58 were defined as primary and 55 as secondary syphilis. A further eight were early latent as defined by previous negative syphilis serology within the past year and no clinical evidence of syphilis.

Before this period, a low incidence of new syphilis cases was diagnosed at the clinics. Six cases were diagnosed in 1997, three cases of latent and three cases of early syphilis; five cases were diagnosed in 1998, two cases of early syphilis. All cases identified in 1999 were latent (27). The cases diagnosed in 1997-9 were in heterosexuals (fig 1). 


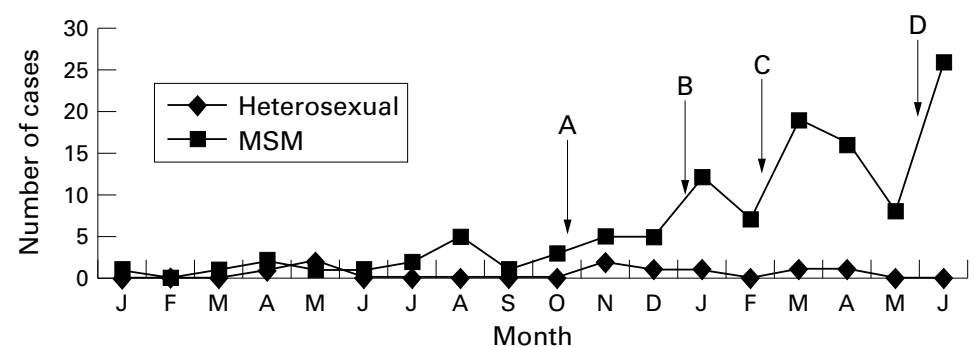

Figure 2 Epigraph of the syphilis outbreak, fanuary 2000-Fune 2001.

EARLY SYPHILIS: JANUARY 2000-JULY 2001

Of the 121 cases, 10 were heterosexual $(8 \%)$ and 111 cases $(92 \%)$ were in men who had sex with men (MSM). The ratio of homosexuals to bisexuals being 98 (88.3\%):13 (11.7\%). The estimated sexually active heterosexual population in Dublin is 500 000. This results in an incidence among heterosexuals of 1 per 100000 in 2000 and 2 per 100000 for 2001 given the current cases. The estimated sexually active population of MSM in the Dublin region is $30000-50000$. The incidence among this population is $46-77$ per 100000 for 2000 and a projected incidence for 2001 of $352-586$ per 100000.

Of the 111 cases in MSM, 51 primary, 52 secondary, and eight early latent cases were diagnosed. The mean age was 35 years (range 19-60 years). One hundred and six were Irish $(95 \%)$. Two cases were from Europe, one case was from the United States, and two cases were from South East Asia. There were 25 HIV positive men in this group $(22.5 \%)$; nine of these were newly diagnosed. The mean length of time since HIV diagnosis in the patients with known HIV infection was 4.6 years (range 2-9 years). Eleven of the $16(68.75 \%)$ known cases were on highly active antiretroviral therapy (HAART). All of these patients had a viral load $<1000 \mathrm{cpm} ; 64 \%$ were below the current limit of detection-that is, $<50 \mathrm{cpm}$. The five patients not on HAART had a mean viral load of $130439 \mathrm{cpm}$ (range 4196-416 $913 \mathrm{cpm}$ ).

The heterosexual cases were diagnosed in four female and six male patients. There were three cases of primary syphilis and one case of secondary syphilis in the female group. The mean age was 32.5 years (range 22-39 years). All three cases of primary syphilis were Irish and were contact traced through their male partners. The case of secondary syphilis occurred in an eastern European female who presented in labour and delivered a stillborn male infant with necropsy findings of congenital syphilis. The six male heterosexual cases were divided into four primary and two secondary cases. The mean age was 30 years. One was contact traced through his female partner. He was also from eastern Europe.

RISK BEHAVIOUR

Among the MSM cases, the mean number of male partners in the past 3 months was 6.5 (range $0-40$ ) and female partners was 0.06 (range 0-3). In the last year, the mean number of male partners for this cohort was 23.6 (range 1-160) and female partners was 0.31 (range 0-12). Among the heterosexuals, four females had one partner each in the preceding 3 months and 1-2 partners in the previous year (mean 1.5). The six heterosexual males had a mean of 1.8 partners in the previous 3 months (range 1-3) and between 1-20 partners in the previous year (mean 5.6, median 2).

Neither heterosexuals nor MSM used condoms for oral sex; however, $49 \%$ of MSM used condoms for penetrative sex in comparison with none of the heterosexuals.

MSM were more likely to use drugs in connection with sexual activities. MSM used alcohol $(89 \%)$, amyl nitrate $(43 \%)$, marijuana $(39 \%)$, Ecstacy $(27 \%)$, and cocaine $(26 \%)$ during or before sexual activity. Only $5 \%$ used heroin at any time in the previous 6 months. Heterosexuals reported only using alcohol $(60 \%)$ and marijuana (10\%) in connection with sexual activity.

SOCIAL NETWORKS

In order to assess possible sites for public health promotion, all positive cases had their sexual and social networks identified. This involved identifying where they met their partners; $95 \%$ of MSM had met partners in the previous 6 months in saunas and clubs $(73 \%$ and $74 \%$ respectively). Only $5 \%$ of MSM had not attended saunas or clubs in the previous 6 months. These cases had exclusively met new partners through friends; $8 \%$ of the total cohort had met partners through internet sites (gaydar.com; gay.com) and $17 \%$ met their partners abroad. Only $1 \%$ met their partners in a park or other outdoor venue; no MSM admitted contact with a commercial sex worker or any links with the industry over the previous year. Heterosexuals met their partners primarily through friends $(75 \%)$. However, $33 \%$ of cases attended clubs regularly. Only one heterosexual met his partners through a sex club/sauna abroad. In total, $22 \%$ met their partners abroad.

SEXUALLY TRANSMITTED INFECTIONS

Forty one per cent of the MSM cases had an additional sexually transmitted infection (excluding HIV) diagnosed; $12 \%$ were codiagnosed with more than one infection. Fifteen $(17 \%)$ were co-diagnosed with Neisseria gonorrhoeae, nine ( $8 \%$ ) with Chlamydia trachomatis, $11(10 \%)$ with non-specific urethritis (NSU), and 11(10\%) with human papillomavirus (clinical genital warts). Four patients $(3.8 \%)$ and one patient $(1 \%)$ were diagnosed with hepatitis $\mathrm{C}$ and hepatitis $\mathrm{B}$ respectively.

Among the HIV positive cases $40 \%$ had an additional STI diagnosed. Four (44\%) of the new HIV cases were co-diagnosed with $N$ gonorrhoeae compared to one of the known cases. Three cases of known HIV were also diagnosed with NSU (18\%) and one with $C$ trachomatis $(6 \%)$.

There was a history of a STI in $36 \%$ of cases in MSM (excluding HIV) with $54 \%$ of cases having attended an STI clinic previously. In comparison, only two (20\%) heterosexual cases had another STI diagnosed, $N$ gonorrhoeae (one) and $C$ trachomatis (one). None of 
the heterosexual cases had previously attended a sexual health clinic.

\section{Discussion}

This increased number of early syphilis cases is of significant concern. Similar trends among MSM have been reported internationally, reflecting a change in sexual behaviour patterns in MSM. ${ }^{5}$ Syphilis is known to increase the transmission rates of HIV and increase HIV viral load. ${ }^{6}$ Sixteen of these men had known HIV and to date there have been a further nine new diagnoses of HIV in this cohort. Significant also in this group was that four patients were also diagnosed with $N$ gonorrhoeae (44\% compared with $11 \%$ in the HIV negative group), an independent risk factor for HIV acquisition. In all, $99.6 \%$ of the cases diagnosed with syphilis have had an initial HIV test performed, however $47 \%$ declined follow up HIV testing at 3 months. Thirty one per cent of the known HIV positive cohort had another STI diagnosed; this reinforces the need for regular sexual health screens and ongoing safer sex messages in this patient group.

Of further concern is the anonymity and number of sexual partners for each case. In the MSM group, over 700 partners were identified in the previous 6 months. However, less than one fifth of these had any contact details. Currently, 103 partners have come forward for testing- $21 \%$ had positive serology, $89 \%$ of this group had evidence of primary or secondary syphilis.

An active educational campaign has been ongoing since January 2001 promoting sexual health screens. Information about syphilis is available throughout the venues where people meet their sexual partners. Advertisements and information was placed on internet sites and in the advertisement section in many magazines and papers.

In addition, on-site testing (healthcare workers offering serological testing) was initiated in
June 2001 in the popular venues (saunas, clubs, and bars) where MSM congregate and meet sexual partners. This has led to a more directed effort by outreach workers and medical staff. As a consequence of this specific intervention, 33 further cases have been identified.

However, as additional cases continue to emerge innovative strategies will continue to be initiated to control this epidemic. Previous studies have shown that contact tracing is an ineffective method of syphilis control. ${ }^{7}$ In small syphilis outbreaks social network tracing that identifies and screens friends of infected individuals have been shown to be as effective as traditional methods. ${ }^{8}$ Further studies into this novel approach may reveal more cases in this population.

Contributors: SH, data collection, analysis and preparation of manuscript; FL, data collection and primary care; CB, supervised collection data and review of manuscript; FM, preparation and review of manuscript.

Financial support: none.

Conflict of interest: none.

1 CDSC. Increased transmission of syphilis in men who have sex with men from Brighton and Hove. Commun Dis CDR Wkly 2000;10:177.

2 CDSC. Increased transmission of syphilis in Manchester. Commun Dis Rep CDR Wkly 2000;10:89.

3 CDC. Outbreak of syphilis among men who have sex with men-southern California, 2000. MMWR Morb Mortal Wkly Rep 2001;50:117-20.

4 CDC. Resurgent bacterial sexually transmitted disease among men who have sex with men - King County, Washington, 1997-1999. MMWR Morb Mortal Wkly Rep 1999; 48:773-7.

5 Stolte IG, Dukers NH, de Wit JB, et al. Increase in sexually transmitted infection among homosexual men in Amsterdam in relation to HAART. Sex Transm Inf 2001;77:184-6.

6 Collis TK, Celum CL. The clinical manifestations and 6 Collis TK, Celum CL. The clinical manifestations and
treatment of sexually transmitted diseases in human immunodeficiency virus-positive men. Clin Infect Dis 2001; 32:611-22.

7 Oxman G, Doyle L. A comparison of the case-finding effectiveness and average cost of screening and partner notification. Sex Transm Dis 1996;23:51-7.

8 Rothenberg RB, Sterk C, Toomey KE, et al. Using social network and ethnographic tools to evaluate syphilis transmission. Sex Transm Dis 1998;25:154-6. 